\title{
Research on the key technologies of intelligent vehicle control
}

\author{
Wenming Cheng \\ Automotive Engineering institute, Jiangxi University of Technology, Nanchang 330098, China
}

Keywords: Intelligent vehicle control; Vehicle; Key technology

\begin{abstract}
The intelligent vehicle, as the key technology of intelligent transportation system, is one of the numerous high-tech integrated carriers. It is a general term that refers to a comprehensive vehicle technology to complete one or many driving tasks entirely or partially. The research work of this paper belongs to the category of intelligent vehicle control technology. Based on our country's automobile traffic situation and technical conditions, the study makes a positive and beneficial exploration to the key technologies of intelligent vehicle control, aiming to provide the necessary technical reserves for the research of the intelligent vehicle in our country and also to provide technical support for the practical application study on China's automobile safety driving assistant system, laying the foundation for the construction of China's intelligent integrated transportation system.
\end{abstract}

\section{Introduction}

From the beginning of the 1970s, the transportation engineers throughout the world have gradually used electronic, information, system engineering and other high-tech means that develop rapidly to improve traffic conditions. Advanced technologies are used to replace partial driving task, providing the automobiles with increasingly perfect driving assistance. The intelligentization of automobiles is gradually achieved.

The intelligent vehicle, as the key technology of intelligent transportation system, is one of the numerous high-tech integrated carriers. It is a general term that refers to a comprehensive vehicle technology to complete one or many driving tasks entirely or partially. The main functions are to ride along the regulated road accurately and maintain on the right lane position, to keep a safe distance between the cars, to adjust the speed according to the traffic conditions and road characteristics, to change lanes and overtake automatically to avoid collision and rear-end accidents, to park the car safely on the urban roads and to let the driver assistant system find the best route to the destination under intelligent traffic network environment, etc. Its existence is a necessity of the development of vehicle technology and the people's urgent demand of traffic safety.

With the continuous progress of the informatization and intelligentization of human society, intelligent vehicles are increasingly widely applied, which has become one of the important symbols to measure a country's social civilization and the progress of science and technology. Intelligent vehicle research began in America in the 1950s. One American company developed the world's first automatic guided vehicle system, and put into operation in the warehouse of the company to realize the automatic transport of goods. USA further studied and improved it, but because of cost and other factors, the application was still confined to the warehouse to transport the goods. But in Europe, the study on intelligent vehicle has developed rapidly. It has been applied to the field of industrial production. The car assembly plant in Sweden adopted the automatic car assembly line based on 
carrying tool, in order to increase the flexibility of the transport system. Since the late eighty's, the research hot spot of intelligent vehicle has been transferred from indoor intelligent vehicle to outdoor. In recent years, in order to reduce traffic accidents, to improve transport efficiency and to reduce the drivers' labor load, America, Britain, Japan, Germany, Canada and other developed countries have carried out the research of intelligent transportation system and intelligent vehicle system. Intelligent vehicle is an important part for it.

Because the traffic system is a very complex system, problems are difficult to solve fundamentally if only considering the vehicle or the road. In this case, the thought of using a variety of high-tech system to solve the traffic problems emerges as the times require, so the western countries put forward the intelligent transportation system. The basic idea is to apply information technology, data communication technology, electronic control technology and computer processing technology comprehensively to the highway transportation system, so as to effectively improve traffic safety, optimize transportation resources, reduce energy consumption and reduce environmental pollution, etc, thus realizing the real intelligentization of transportation system. In fact, the development of intelligent transportation system promotes the second rapid development of intelligent vehicle.

At present, the research of intelligent vehicle mainly includes the following aspects.

(1) The drivers' behavior analysis mainly studies the intrinsic link between driver's behavior and mental state and vehicle riding, aiming to establish a variety of assistant driving models to provide necessary data for the intelligent vehicle safety driving assistant or automatic driving. For example, the classified analysis of the driver's facial expressions can determine whether the driver is fatigue or sleepy, etc.

(2) Environmental perception is mainly to use sensor fusion technology to obtain useful information of vehicle driving environment, such as traffic information, lane status information, the velocity information of the surrounding vehicles and traffic sign information, etc.

(3) Autonomous driving in extreme cases mainly researches the vehicle autonomous driving in some extreme cases, such as reaction limit, driver losing control of the vehicle, etc.

(4) Autonomous navigation under the norm environment mainly researches the autonomous completion of vehicle driving behavior in some standard conditions. For example, if there are human-set signs or better road conditions, intelligent vehicle combines the control model with environmental data obtained based on the perception of the environment to complete autonomous driving without human intervention.

(5) The vehicle motion control system studies kinematics of vehicle control, dynamics modeling and vehicle body control, etc.

(6) Compared with passive safety system, active safety system focus on prevention, for example, studies the obstacle avoidance, anti-collision safety system in various cases.

(7) Traffic monitoring and vehicle navigation and coordination mainly study traffic flow guidance, etc.

(8) The vehicle interaction and communication studies the exchange of information between the vehicle, mainly focusing on the problem of wireless communication between various vehicles

(9) The military application researches the application of intelligent vehicle system in the military

(10) Systematic structure studies the structure and organization issues of the intelligent vehicle system. 
(11) Advanced vehicle security researches the vehicle system with higher safety and more intelligent features.

The research work of this paper belongs to the category of the control technology of intelligent vehicle. This study is based on China's automobile traffic situation and technical conditions, carries out a positive and beneficial exploration to the key technology of intelligent vehicle control, aiming to provide the necessary technical reserves for the intelligent vehicle and its research in our country. It also provides practical technical support for the application research on China's automobile safety driving assistant system, laying the foundation for the construction of China's intelligent integrated transportation system.

\section{Methods}

The control system is the "brain" of intelligent vehicle, reflecting the intelligence degree of the autonomous controller and organizational flexibility.

With the rapid development of modern science and technology such as computers, materials, energy and others and the unceasing expansion of the production system, a complex control system has been formed, contributing to more complicated control objects, controller and control tasks, etc. At the same time, there are more requirements about the degree of automation, facing the challenges of the complex systems including the flexibility control system, intelligent robot system, CNC system, computer integrated manufacturing system and others. The classical and modern control theory and technology is no longer suitable for the control of complex systems.

Intelligent control is a kind of advanced information and control technology which is gradually formed on the basis of control theory, information theory, artificial intelligence, bionics, neurophysiology and the development of computer science. Intelligent control theory breaks through the traditional control framework based on the mathematical model. It basically controls according to the actual effect, which is not dependent on or not entirely dependent on the mathematical model of the control object, but also inherits the nonlinear characteristics of human thinking. Some intelligent control methods also have functions including online identification and decision-making or overall self optimizing ability and the processing and decision of hierarchical information.

The expert control is to imitate experts' intelligence to realize the effective control of the system in an unknown environment, based on the combination of the theory and technology in the expert system with the control theory and methods. According to the complexity of the application of expert system technology in the control system, it can mainly be divided into expert control system and expert controller. The expert control system has comprehensive expert system structure, sophisticated and reliable knowledge processing and real-time control function, huge knowledge base and complex inference mechanism. Expert controller mostly is industrial expert controller with small knowledge base and simple inference mechanism, which focuses on heuristic control of the development of knowledge. It has real-time algorithm and logic function. The core of expert control is the expert system which has a variety of non-structural problems. Especially when processing qualitative, heuristic or uncertain knowledge and information, we must go through various inference processes to achieve the systematic control targets.

Normal cloud is the most important cloud model, which is most useful to express language value, because each branch of social and natural science has proved the universality of normal distribution. The mathematical expectation curve of the normal cloud is: 


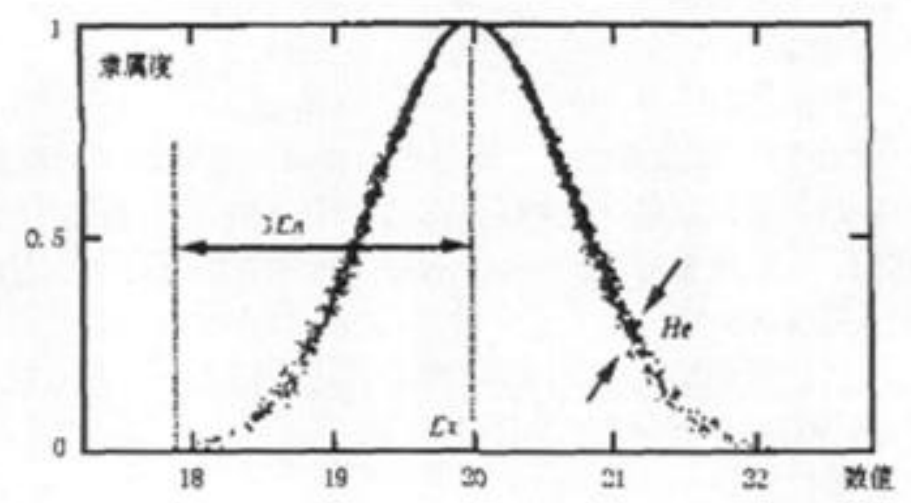

Fig. 1 one-dimension normal descriptive graph

Intelligent vehicle, as the key technology of intelligent transportation system, is a comprehensive system integrating environmental perception, planning and decision-making and multi-scale assistant driving and other functions. It focused on the application of computers, modern sensing, information fusion, communication, artificial intelligence and automatic control technology, which is a typical high-tech complex. It has the road obstacles automatic identification, automatic alarm, automatic braking, automatic maintenance of a safe distance, speed and cruise control and other functions.

In the research of intelligent vehicle, autonomous driving and aided navigation are the key technologies of intelligent vehicle. From the history of intelligent vehicle development, a variety of modes of autonomous driving and aided navigation have emerged, including underground wire-embedded navigation, optics navigation, visual navigation, ultrasonic navigation and infrared navigation, etc.

In the intelligent highway system, the study on vehicle lateral control is the basis for intelligent vehicle control. Vehicle lateral control is to control the vehicle by using the signal detected by vehicle sensors and processed by the vehicle under the guidance of some navigation system, making the vehicle able to drive automatically in the specific driveway, and finally realizing the automatic driving of the vehicle.

Once the driver determines the expected position, he will instinctively try to reach the target position following an imaginary a route. Here we simulate human's dynamic thinking process that will change continuously with time and can sense the environmental constraints Therefore, generating and following the modeling route of the driver's route is a continuous process. That is to say, in the simulation of vehicle going from the current position to the dynamic target position, the vehicle transforms the coordinates unceasingly and calculates the route, with the sampling time as the interval.

Lane change contains the generation of lane change intention, its feasibility analysis and the implementation of lane changing behavior. Mandatory lane changing refers to the lane changing behavior that must be taken by the vehicle in order to complete the normal driving purpose. The three most common mandatory lane changing cases are given in the graph. Because the vehicle ahead is parking and blocks the left turn for continuing driving on the current lane, the vehicle prepares to change lane leftwards when it drives on the entrance lane of the intersection and has seen the lane guiding markings ahead. The vehicle is close to the end of the current lane, so it will be ready to change lanes to the left. In addition, the lane changing behavior of the bus when it approaches the front stop and changes its lane from the inside lane to the outside of the dock lane is also a common mandatory lane changing behavior. 
Obstacle avoidance is one of the key problems reflecting the autonomous navigation ability of intelligent vehicle. Obstacle avoidance in the dynamic environment is very difficult and must be resolved, so many scholars have studied such kind of collision avoidance problem and put forward some methods. At present, the achievements of researches on dynamic obstacle avoidance problem in the field of intelligent vehicle are very few, mainly focusing on using three-layer fuzzy method to control the speed and movement direction of robot in the field of robot dynamic obstacle avoidance, realizing dynamic obstacle avoidance. Based on the path planning of fuzzy logic, Xie Jing introduced the collision risk degree as the basis of decision control to improve the gravity function and repulsion function in the traditional artificial potential field method and added the relative velocity parameters of the obstacles and robot to the functions. Dong Lizhi used minimum variance prediction algorithm to estimate the obstacle position of the next time, thus including the motion information of the obstacle in the traditional artificial potential field method. Yuan Cengren improved and combined grid method and regression method. Zhuang Xiaodong put forward a dynamic environmental model based on fuzzy concept and brought about concepts and methods of velocity obstacle and velocity potential field. Chen Huahua put forward a dynamic obstacle avoidance algorithm based on neural network and genetic algorithm and used space and time coordinate to transfer $2 \mathrm{D}$ dynamic environment into three-dimensional static environment in space which is transferred into the obstacle avoidance problem in static environment. Zhang Feng put forward a dynamic obstacle avoidance algorithm based on relative coordinates in the acceleration space. Duan Hua proposed a dynamic obstacle avoidance approach based on the maximum bounding box section. Duan Hua also presented a dynamic obstacle avoidance method based on potential field theory.

From the beginning of 1970s, transportation engineers throughout the world gradually use the rapid developed electronic, information, system engineering and other high-tech means to improve traffic conditions and attempt to complete the driving task by replacing human with intelligent vehicle so as to solve the problem of traffic safety. But in the long-term study process, there are still more technical difficulties in entirely replacing human's driving with intelligent vehicle, so people put forward a safety driving assistant from the perspective of being practical and safe. The lane changing and overtaking of the vehicle are common operation of the driver's, which is the process of the driver's comprehensive behavior where corresponding to the stimulation of the information of surroundings such as the speed of surrounding vehicles, the distance between vehicles, etc, the driver adjust and complete the driving target strategy. In such a complicated process, the longitudinal control and vehicle lateral control of the vehicle must be taken into account as well as the longitudinal control of lane change and overtaking at the same time. Researches on the lane changing and overtaking is relatively few, mainly concentrating on the study of control for the auxiliary safety system of vehicle lane changing and simplified lane changing control. Assuming that when the vehicle changes lane, the transverse velocity maintains the constant speed, the traditional two degrees of freedom vehicle dynamics model is used and optimal control theory is applied to design optimal controller in order to conduct simulation analysis for the lane changing of different speeds. This paper puts forward a method where when the lane is changed, the vehicle longitudinal velocity remains the same. This method treats the lane charging control as single lateral control to simplify the design of lane changing controller. The lane changing track is formed by integrating predefined the lateral acceleration. Construct two degrees of freedom control model of the vehicle and design sliding mode controller based on yaw rate. A vehicle lateral control algorithm for each vehicle in the queue is designed. Verify the possibility of using fuzzy controller for vehicle automatic control. 
There is a total control rules, all based on drivers' experience. The controller has three input, namely, the transverse displacement of the vehicle, the current lateral acceleration of the vehicle and the difference between the current lateral acceleration and the expected lateral acceleration. The input of the controller is the front wheel steering angle. First, get the environmental information. Then, quickly plan the method of the vehicle lane changing track and overtaking track. Finally, use modern control theory to design tracking controller of lane changing and overtaking track, achieving the function of automatic overtaking and automatic lane changing of the intelligent vehicle. Based on the analysis and summary of the above methods, the paper proposes a novel and effective method of the automatic lane change and automatic overtaking. By establishing the concept of dynamic target position and putting forward the control mechanism based on it, it designs controller to control the vehicle intelligently, effectively realizing the automatic lane changing and overtaking of vehicle.

\section{Conclusion}

Intelligent vehicle is a research frontier and hot spot in the field of vehicle engineering in the world. Based on the scientific research and technology conditions in our country, the paper implements a systematic research on the key technology of intelligent vehicle control. The main work of this paper is to put forward an inference model by combining cloud theory, inspired by fuzzy model. The model softens and divides the whole area into several cloud sub areas. The corresponding input of each cloud sub area is the linear expression of input value. The belonging degree of the input value to some cloud sub area is considered as the random confidence of the rule. The characteristics of the entire nonlinear system are the weighting sum of the local linear models, which not only inherits the success of cloud theory but also can overcome the shortcomings of the traditional inference mechanism based on cloud model. It has strong function approximation ability that can be used for system identification, intelligent control and other fields.

Forward cloud is generated by using the golden ratio method to make the concept of expression more reasonable. The inference model is treated as the reasoning mechanism of the controller, softening and dividing the entire area into several sub areas. In the corresponding sub areas the use of radial basis function neural network approximation equation of the driving can make the controller have learning function, well simulating the driver's learning process. The area neural network output value corresponding to each cloud sub area is the output of this area. The belonging degree of the output value to some cloud sub area is considered as the random confidence of the rule. The random output of the entire nonlinear system is the weighting sum of the local random output which can identify the nonlinear system quite well.

Because of the time constraints of objective conditions, many studies have not yet completed. Based on the current research results, as for the problems involved in the study, we make the following expectations. In the paper, in the part of designing intelligent controller design algorithm based on RBF neural network, due to the limitation of the concept of cloud generation algorithm based on the golden ratio, the entropy is determined by people, so it is not objective. It is seeking for a method to solve this problem--- the rationality of the concept generation and the accuracy of control. The research of intelligent vehicle control based on dynamic target position studies transmission control of intelligent vehicle, but its speed is mechanical and cannot fully simulate the driver's acceleration and deceleration driving behavior, driver. It is expected to study the dynamic target position control mechanism based on the simulation of driving speed. The selection of the dynamic target position in intelligent vehicle control in this paper is too ideal and there is a certain 
gap with reality, which cannot completely simulate the driving decision behavior of the driver, so we try to study the path planning of intelligent vehicle dynamic target position control mechanism. The strategy of intelligent traffic signal control based on cloud theory is based on the single intersection. In reality, we should consider the multi-intersection system coordination control strategy and try to study multi-intersection coordination control optimization strategy based on cloud theory.

Intelligent vehicle is one of the world's cutting-edge researches in the field of vehicle engineering. The prospective exploration and the basic research about it, on the one hand, can be used as an important technical reserve, contributing to seizing the future technology, which has the important strategic status and research value. On the other hand, many mature relevant technologies can be applied to the existing vehicle to provide safe auxiliary driving function for the driver, which will have significant social and economic benefits. As for the intelligent vehicle system, the content of this paper is just a very small part of the whole research. In the future study and work, it will be dedicated to the related theoretical research and application development, making it more intelligent and practical.

\section{Acknowledgements}

This work was financially supported by the key subject building project (vehicle engineering) of Jiangxi University of Technology.

\section{References}

[1] Bishop R. A survey of intelligent vehicle applications worldwide[C]//Intelligent Vehicles Symposium, 2000. IV 2000. Proceedings of the IEEE. IEEE, 2000: 25-30.

[2] Reichardt D, Miglietta M, Moretti L, et al. CarTALK 2000: Safe and comfortable driving based upon inter-vehicle-communication[C]//Intelligent Vehicle Symposium, 2002. IEEE. IEEE, 2002, 2: 545-550.

[3] Vahidi A, Eskandarian A. Research advances in intelligent collision avoidance and adaptive cruise control[J]. Intelligent Transportation Systems, IEEE Transactions on, 2003, 4(3): 143-153.

[4] Tsugawa S. Inter-vehicle communications and their applications to intelligent vehicles: an overview[C]//Intelligent Vehicle Symposium, 2002. IEEE. IEEE, 2002, 2: 564-569.

[5] Xiwen L, Yinggang O, Zuoxi Z, et al. Research and development of intelligent flexible chassis for precision farming[J]. Transactions of The Chinese Society of Agricultural Engineering, 2005, 2: 019.

[6] Qian Y, Moayeri N. Design of secure and application-oriented VANETs[C]//Vehicular Technology Conference, 2008. VTC Spring 2008. IEEE. IEEE, 2008: 2794-2799.

[7] Luo J, Hubaux J P. A survey of research in inter-vehicle communications[M]//Embedded Security in Cars. Springer Berlin Heidelberg, 2006: 111-122.

[8] Liu Q, Lu H, Zhang H, et al. Research and design of intelligent vehicle monitoring system based on GPS/GSM[C]//ITS Telecommunications Proceedings, 2006 6th International Conference on. IEEE, 2006: 1267-1270. 
[9] Giannopoulos G A. The application of information and communication technologies in transport[J]. European Journal of Operational Research, 2004, 152(2): 302-320.

[10]Bertozzi M, Broggi A, Fascioli A. Vision-based intelligent vehicles: State of the art and perspectives[J]. Robotics and Autonomous systems, 2000, 32(1): 1-16. 